\title{
Sensorkonzept zur in vitro Echtzeitmessung des Degradationsverhaltens von biodegradierbaren Polymeren
}

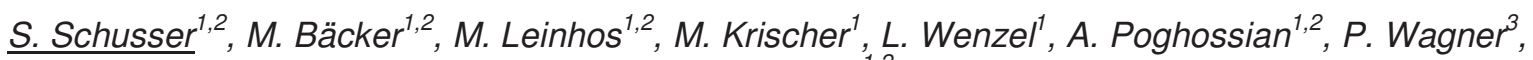 \\ M.J. Schöning ${ }^{1,2}$
}

${ }^{1}$ Institut für Nano- und Biotechnologien (INB), FH Aachen, Campus Jülich, Deutschland

schusser@fh-aachen.de

${ }^{2}$ Peter Grünberg Institut (PGI-8), Forschungszentrum Jülich GmbH, Deutschland

${ }^{3}$ Instituut voor Materiaalonderzoek (IMO), Universiteit Hasselt, Belgien

\begin{abstract}
Kurzfassung:
In vitro Untersuchungen des Degradationsverlaufs sind essentiell für die Entwicklung und Optimierung implantierbarer Medizinprodukte auf der Basis von biodegradierbaren Polymeren. Erstmals wurde mit Hilfe eines halbleiterbasierten kapazitiven Feldeffektsensors, der über Impedanzspektren ausgelesen wird, ein Sensorsystem realisiert, das die Echtzeitmessung der Degradation in situ ermöglicht. In einem Modellversuch wurde die Funktionalität des System demonstriert, indem die Degradation eines kommerziell erhältlichen, biodegradierbaren Polymers (Poly-D,L-Milchsäure) in Echtzeit verfolgt wurde. Dazu wurde das Polymer mittels Spin-Coating auf den Sensor aufgebracht und zunächst einer neutralen Pufferlösung ( $\mathrm{pH} 7$ ) zur Degradation ausgesetzt. Nach einer Zeit von 53,5 h wurde der pH-Wert auf $\mathrm{pH} 9$ erhöht, um die hydrolytische Degradation zu beschleunigen. Die derart erzeugte Veränderung der Degradationsrate des Polymers konnte in einer beschleunigten Abnahme der Impedanzwerte des Sensorsignals gezeigt werden.
\end{abstract}

Keywords: Impedanzspektroskopie, (bio)degradierbare Polymere, Elektrolyt-Isolator-HalbleiterSensor (EIS-Sensor), Echtzeit-Degradationsmessung, Polymilchsäure

\section{Einleitung}

Biodegradierbare Polymere spielen in biomedizintechnischen Anwendungen bereits jetzt eine besondere Rolle. Ihre Eigenschaft, sich unter physiologischen Bedingungen aufzulösen, eröffnet neue Möglichkeiten für die Entwicklung von funktionalen Produkten und hat bereits zu einem breiten Spektrum an Anwendungsszenarien geführt, die diese besondere Fähigkeit ausnutzen. So wurden bereits Gerüste zur Gewebezüchtung [1], Wundverbände [2], orthopädische und dentale Implantate [3] und Wirkstofffreisetzungs-Systeme (Drug-Delivery-Systeme) [4] in der Literatur beschrieben, die die be- die beschränkte Lebensdauer eines Polymers gezielt nutzen, um die Funktionalität zu erweitern.

Bisher beschränkt sich die Auswahl an existierenden Materialien, die für die medizinische Nutzung freigegeben wurden, auf eine geringe Anzahl von Polymeren [2] und selbst deren physiologische Verträglichkeit wird in der Literatur teilweise kontrovers diskutiert [5]. Mit Hinblick auf das Potential dieser Materialien ist allerdings zu erwarten, dass in den kommenden Jahren neue Polymere entwickelt werden, die die hohen Anforderungen von Seiten der physiologischen Verträglichkeit aber auch der un- 
terschiedlichen Anwendung an das Material, besser gerecht werden.

Eine Herausforderung bei der Entwicklung ist dabei der Zeitbereich, über den das Polymer degradieren soll. Aufgrund der Abhängigkeit von diversen Faktoren wie z.B. dem pH-Wert, der Temperatur und der lonenstärke des umgebenden Mediums, ist eine Vielzahl von Untersuchungen nötig, um diesen Aspekt in ausreichendem Umfang zu validieren.

Der in dieser Arbeit vorgestellte Ansatz beschreibt daher ein Sensorkonzept, das zur Echtzeitüberwachung des Degradationsverlaufs dient und den Aufwand von Degradationsstudien, die zur Validierung aber auch Optimierung des Degradationsverhaltens von Polymeren notwendig sind, reduzieren soll. Zum Einsatz kommt dafür ein halbleiterbasierter kapazitiver Feldeffektsensor, der mittels Impedanzspektroskopie ausgelesen wird und so zerstörungsfrei ein zeitlich aufgelöstes Signal ermittelt, das Aufschluss über den Degradationsverlauf gibt. Die dadurch erreichte Zeitauflösung wäre unter Verwendung der bislang überwiegend verwendeten, zerstörungsbehafteten Messmethoden nur unter Einsatz einer unverhältnismäßigen Anzahl an Proben zu erreichen, was für die Umsetzung einen nicht praktikablen Aufwand bedeutet.

Exemplarisch wurde das hier vorgestellte Sensorkonzept eingesetzt, um die Abhängigkeit der Degradationskinetik eines kommerziell erhältlichen Polymers auf der Basis von Poly-D,LMilchsäure (PDLLA) vom umgebenden Medium zu untersuchen und im speziellen die Abhängigkeit vom $\mathrm{pH}$-Wert aufzuzeigen.

\section{Sensorprinzip und Herstellung}

Abbildung 1 zeigt den grundlegenden Aufbau a)

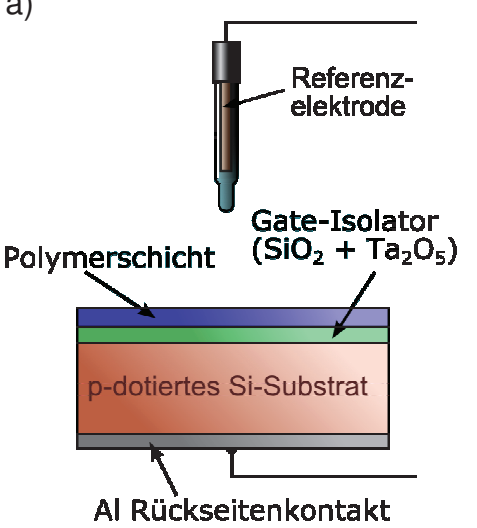

b)

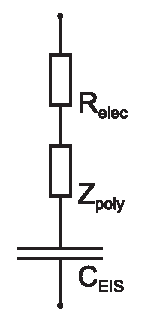

Abb. 1. Schichtaufbau des Sensors (a) und idealisiertes Ersatzschaltbild der Sensorstruktur (b) bestehend aus der kombinierten Kapazität der Halbleiterstruktur und Isolatorschicht $C_{E I S}$, einem nicht spezifizierten Impedanzelement zur Beschreibung der Polymerschicht $Z_{\text {poly }}$ und dem Elektrolytwiderstand $R_{\text {elec. }}$ der Sensorstruktur der verwendeten ElektrolytIsolator-Halbleiter-Sensoren (EIS-Sensoren) und deren idealisierte Darstellung im elektrischen Ersatzschaltbild. Der EIS-Sensor besteht aus einem p-dotiertem Siliziumsubstrat mit einer typischen Dicke von ca. $450 \mu \mathrm{m}$ und einer Fläche von $10 \mathrm{~mm} \times 10 \mathrm{~mm}$, die durch ein isolierendes Gate (hier eine Schicht aus $30 \mathrm{~nm}$ $\mathrm{SiO}_{2}$ und $60 \mathrm{~nm} \mathrm{Ta}_{2} \mathrm{O}_{5}$ ) bedeckt ist. Auf dieses Gate ist das zu untersuchende Polymer als dünne Schicht aufgebracht, die dem Degradationsmedium ausgesetzt wird. Über eine $\mathrm{Ag} / \mathrm{AgCl}$-Referenzelektrode wird das Degradationsmedium elektrisch mit einem Impedanzanalysator (IM6, Zahner Elektrik GmbH \& CoKG, Germany) verbunden, der auf der Gegenseite über einen ohmschen Kontakt (Schicht aus $300 \mathrm{~nm} \mathrm{Al}$ auf der Substratrückseite) mit dem Siliziumsubstrat des Sensors verbunden ist. Jedwede Änderung der elektrochemischen Eigenschaften des Polymers, die durch die Degradation verursacht wird, kann so als Änderung der Impedanz erfasst werden und Auskunft über das Fortschreiten des Degradationsprozesses geben.

Im vorliegenden Versuch wurde die Poly-D,LMilchsäure (PDLLA) RESOMER ${ }^{\circledR}$ R 202 H (Evonik Röhm $\mathrm{GmbH}$, Deutschland) verwendet, die in Aceton gelöst und mittels Spin-Coating als definierte dünne Schicht von 500 nm Dicke auf den Chip aufgebracht wurde.

\section{Messaufbau und Durchführung}

Der beladene Sensor wurde in eine Messzelle

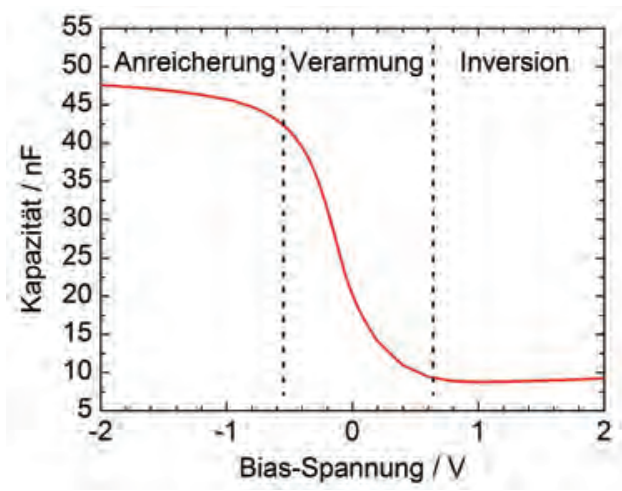

Abb. 2. Verdeutlichung der Zustände eines halbleiterbasierten kapazitiven Feldeffektsensors und die daraus resultierende Kapazität (gezeigt ist der Hochfrequenzfall): Für niedrige Spannungen befindet sich der Sensor im Anreicherungszustand und die Kapazität wird maßgeblich durch die Isolatorschicht bestimmt. Mit zunehmender Bias-Spannung werden die Majoritätsladungsträger aus dem angrenzenden Bereich zur Isolatorschicht verdrängt und es bildet sich eine Raumladungszone aus, die die Gesamtkapazität verringert (Verarmung). Mit weiter anwachsender Vorspannung tritt die Inversion des Halbleiters am Übergang zum Isolator auf, die einer weiteren Vergrößerung der Raumladungszone entgegenwirkt und die Gesamtkapazität einem konstanten Wert entgegen streben lässt. 
eingebaut und mit einem auf die Sensoroberfläche aufgedrückten O-Ring abgedichtet. Die aktive Sensorfläche, die in Kontakt zum Degradationsmedium steht, betrug dadurch ungefähr $0,5 \mathrm{~cm}^{2}$

Mittels Impedanzspektren wurde die Sensorimpedanz in regelmäßigen, zeitlichen Abständen ausgelesen. Dafür wurde eine kleine Wechselspannung von $20 \mathrm{mV}$ mit veränderlicher Frequenz zwischen $0,1 \mathrm{~Hz}$ und $1 \mathrm{kHz}$ eingeprägt, die einer statischen Bias-Spannung von -2 V überlagert wurde. Letztere wird benötigt, um das Halbleitermaterial des Sensors vorzuspannen und so den gewünschten Zustand des Sensors einzustellen. Abbildung 2

a)

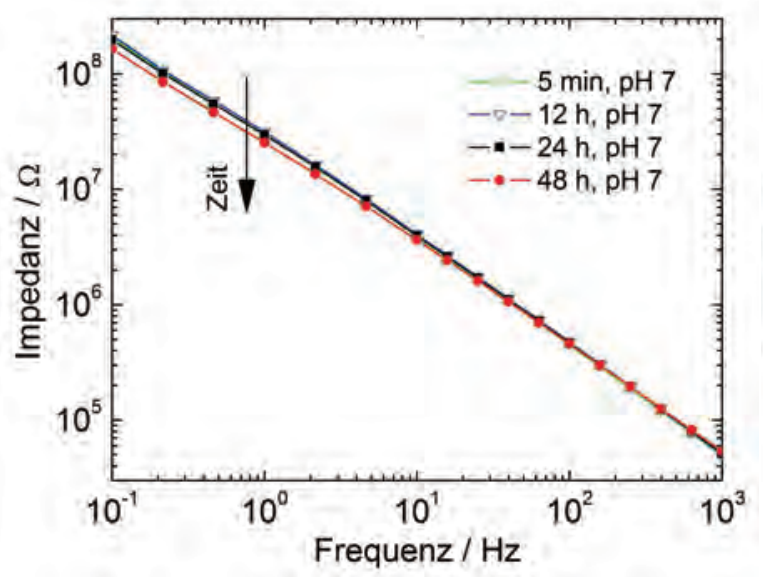

Degradationrate. Das wird auch aus den Impedanzspektren aus Abb. 3 a ersichtlich. Erst nach $48 \mathrm{~h}$ in Kontakt zum Degradationsmedium zeigen sich messbare Veränderungen der Impedanzwerte. Anders verhält sich das Polymer dagegen in alkalischen Medien (Abb. 3 b). Bereits nach $12 \mathrm{~h}$ zeigen sich vor allem im Frequenzbereich unterhalb von $100 \mathrm{~Hz}$ Abnahmen von bis zu etwa einer halben Dekade, die im weiteren Verlauf weiter fortschreiten.

\section{Zusammenfassung}

Die Untersuchung von Degradationsprozessen (bio)degradierbarer Polymere unter der Verwendung der üblichen Offline-Analysen erfor-

b)

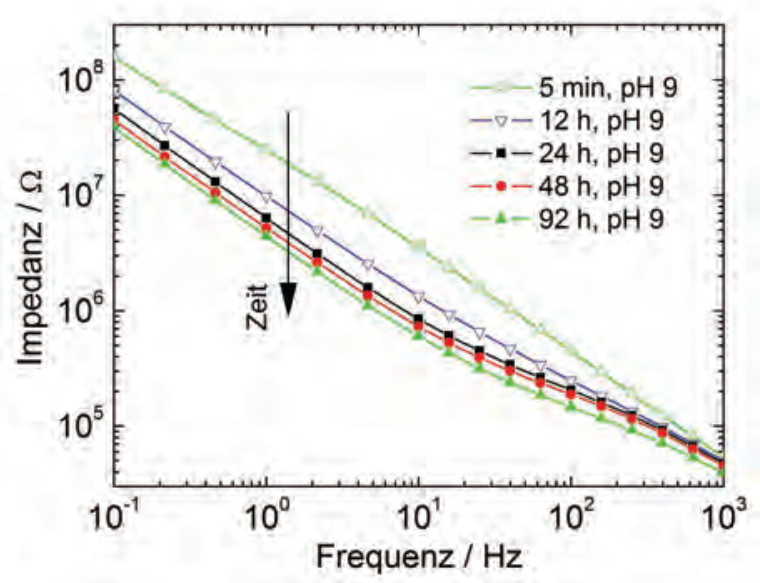

Abb. 3. Impedanzspektren der Sensorstruktur mit degradierender Polymerschicht aus PDLLA, nachdem sie bestimmte Zeiten den Puffermedien von $\mathrm{pH} 7$ (a) und pH 9 (b) ausgesetzt war.

zeigt den Zusammenhang zwischen eingeprägter Bias-Spannung und der resultierenden Kapazität des EIS-Sensors. Weitere Details zum Funktionsprinzip und der Physik dieser Art von Sensoren sind in [6] zu finden.

In der ersten Phase des Versuchs wurde der Sensor mit Polymer für 53,5 h einer Pufferlösung mit pH 7 ausgesetzt. Danach wurde der $\mathrm{pH}-$ Wert des Mediums auf $\mathrm{pH} 9$ erhöht. Da PDLLA durch Hydrolyse der Esterbindung zwischen den Monomeren degradiert und dieser Vorgang stark vom umgebenden $\mathrm{pH}$-Wert abhängig ist, erzeugt der hier induzierte Anstieg des $\mathrm{pH}$-Werts eine beschleunigte Degradation des PDLLAs [7].

\section{Ergebnisse}

Abbildung 3 zeigt ausgewählte Impedanzspektren des Degradationsverlaufs von PDLLA zu bestimmten Zeitpunkten. Wie aus der Literatur bekannt, zeigt PDLLA in Umgebungen mit neutralem $\mathrm{pH}$ eine verhältnismäßig langsame dert einen erheblichen Aufwand in der Durchführung, um eine adäquate Zeitauflösung zu gewährleisten. Der Ansatz einer in vitro Überwachung mittels impedanzspektroskopischer Analysen, wie die hier gezeigte Untersuchung mittels halbleiterbasierten kapazitiven Feldeffektsensoren, stellt daher eine einfache und vielversprechende Methode dar, um den Aufwand von Untersuchungen solcher Polymere zu reduzieren. Anhand beispielhafter Messungen wurde das Degradationsverhalten von PDLLA in $\mathrm{pH}$-neutralem Medium und nach Alkalisierung in Echtzeit verfolgt, und es konnte die beschleunigte hydrolytische Degradation des Polymers durch eine ebenfalls schnellere Änderung der gemessenen Impedanzwerte gezeigt werden. Diese erfolgreichen, ersten Experimente demonstrieren das Potential dieses Ansatzes für die in situ Echtzeitmessung von Degradationsvorgängen biodegradierbarer Polymere. Gegenstand zukünftiger Arbeiten stellt darüber hinaus die Erarbeitung von detaillierten elektrischen Ersatzschaltbildern dar, um tiefergreifen- 
dere Information über den Degradationsverlauf aus den in den Impedanzspektren enthaltenden Daten zu ziehen.

\section{Danksagung}

Die Durchführung dieser Arbeit fand im Rahmen des Interreg EMR IV-A Projektes BioMIMedics (www.biomimedics.org) statt und wurde von der Europäischen Union, regionalen Behörden, Forschungsinstituten und KMUs kofinanziert. Die Europäische Kommission investiert in Ihre Zukunft.

\section{Referenzen}

[1] M. Martina, D. W. Hutmacher, Biodegradable polymers applied in tissue engineering research: a review, Polymer International 56, 145-157 (2007); doi: 10.1002/pi.2108

[2] L. S. Nair, C. T. Laurencin, Biodegradable polymers as biomaterials, Progress in Polymer Science 32, 762-798 (2007); doi: 10.1016/j.progpolymsci.2007.05.017

[3] W. Amass, A. Amass, B. Tighe, A review of biodegradable polymers: uses, current developments in the synthesis and characterization of biodegradable polyesters, blends of biodegradable polymers and recent advances in biodegradation studies, Polymer International 47, 89-144 (1998); doi: 10.1002/(SICI)1097-

0126(1998100)47:2<89::AID-PI86>3.0.CO;2-F

[4] C. Engineer, J. Parikh, A. Raval, Review on hydrolytic degradation behavior of biodegradable polymers from controlled drug delivery system, Trends in Biomaterials and Artificial Organs 25, 79-85 (2011);

[5] K. A. Athanasiou, G. G. Niederauer, C. Agrawal, Sterilization, toxicity, biocompatibility and clinical applications of polylactic acid/ polyglycolic acid copolymers, Biomaterials 17, 93-102 (1996); doi: 10.1016/0142-9612(96)85754-1

[6] A. Poghossian, M.J. Schöning, Silicon-based chemical and biological field-effect sensors, Encyclopedia of Sensors, vol. 9, American Scientific Publisher, Santa Clarita, California, USA, pp. 463-534 (2006)

[7] F. von Burkersroda, L. Schedl, A. Göpferich, Why degradable polymers undergo surface erosion or bulk erosion, Biomaterials 23, 4221-4231 (2002); doi: 10.1016/S0142-9612(02)00170-9 